\title{
Extracellular volume fraction correlates with myocardial stiffness and allows for differentiation between impaired active relaxation and passive stiffness in heart failure with preserved ejection fraction
}

\author{
Karl-Philipp Rommel", Maximilian von Roeder, Thomas Stiermaier, Konrad Latuscynski, Christian Oberueck, \\ Stephan Blazek, Marcus Sandri, Christian F Luecke, Matthias Gutberlet, Gerhard Schuler, Philipp Lurz
}

From 19th Annual SCMR Scientific Sessions

Los Angeles, CA, USA. 27-30 January 2016

\section{Background}

Optimal patients characterization in Heart Failure with Preserved Ejection Fraction (HFpEF) is essential to tailor successful treatment strategies.

Cardiac magnetic resonance derived T1-Mapping allows for non-invasive quantification of diffuse myocardial fibrosis as extracellular volume fraction (ECV).

We aimed to elucidate the diagnostic performance of T1-Mapping in HFpEF by examining the relationship between ECV and invasively measured parameters of diastolic function and investigated the potential of ECV to differentiate between different pathomechanisms in HFpEF.

\section{Methods}

We performed T1-Mapping in 21 patients with HFpEF and 11 patients without heart failure symptoms. Pressure-volume-loops were obtained with a conductance catheter during basal conditions and handgrip exercise. Transient preload reduction was used to extrapolate the diastolic stiffness constant.

\section{Results}

Patients with HFpEF showed a higher ECV ( $p=0.001)$, an elevated load-independent passive LV-stiffnessconstant $\beta(\mathrm{p}<0.001)$ and a longer time constant of active LV-relaxation $\tau(\mathrm{p}=0.04)$. ECV correlated highly with $\beta(\mathrm{r}=0.75, \mathrm{p}<0.001)$. After multivariate analysis, ECV remained the only independent predictor of $\beta$.

Within the HFpEF cohort, patients with ECV > median showed a higher LV-stiffness-constant $(\mathrm{p}=0.05)$ whereas $\mathrm{ECV}<$ median identified patients with a prolonged active LV-relaxation $(\mathrm{p}=0.01)$ and a marked hypertensive reaction to exercise due to a pathologic arterial elastance $(\mathrm{p}=0.05)$.

\section{Conclusions}

Diffuse myocardial fibrosis, assessed by CMR derived T1-Mapping, independently predicts invasively measured LV stiffness in HFpEF. In addition, ECV helps to noninvasively distinguish the role of impaired active relaxation and passive stiffness. It also refines characterization of patients, which represents a prerequisite for any successful therapy in the future.

Published: 27 January 2016

doi:10.1186/1532-429X-18-S1-Q66

Cite this article as: Rommel et al:: Extracellular volume fraction

correlates with myocardial stiffness and allows for differentiation between impaired active relaxation and passive stiffness in heart failure with preserved ejection fraction. Journal of Cardiovascular Magnetic Resonance 2016 18(Suppl 1):Q66. 

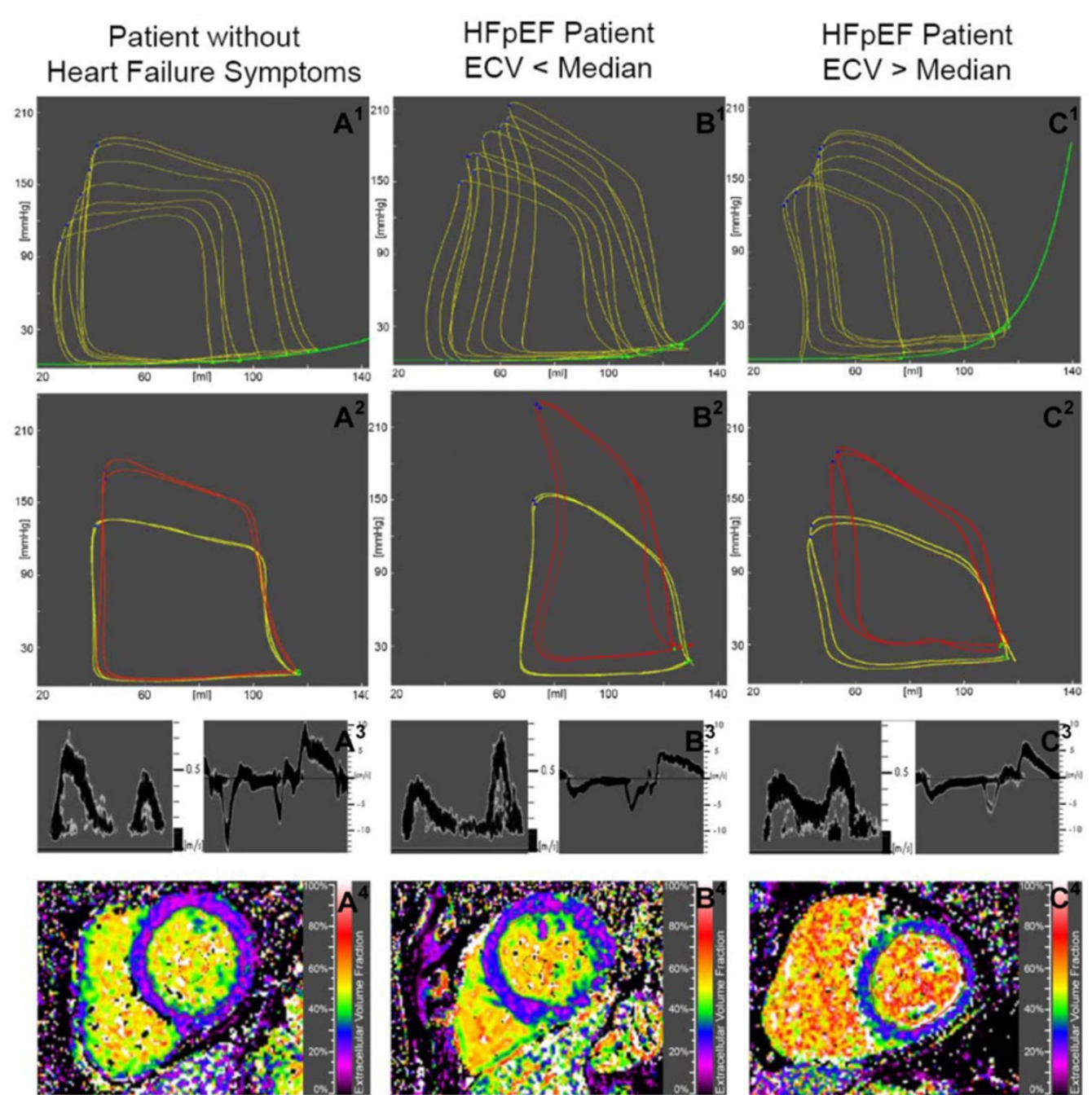

(A) Patient without HF symptoms 1: Pressure-volume loops under transient preload reduction show a shallow slope of the EDPVR line (green line). 2: Pressure-volume loops at baseline (yellow) and throughout exercise (red) with physiologic response to exertion and a small change in EDPVR (green points) 3 : Echocardiographic assessment of MV inflow pattern (left) and septal MV annular velocities (right) with no evidence of diastolic dysfunction. 4) CMR-derived T1-Mapping with native T1-Map (left) and ECV-Map for 3 slices (right) demonstrating a low ECV of $25 \%$.

(B) HFpEF patients with ECV < Median (B) 1: Pressure-volume loops under transient preload reduction shows an increased slope of the EDPVR line (green line). 2: Pressure-volume loops at baseline (yellow) and throughout exercise (red) with elevated arterial elastance and marked hypertensive response to exertion resulting in significant change in EDPVR (green points) 3: Echocardiographic assessment of MV inflow pattern (left) and septal MV annular velocities (right) shows diastolic dysfunction. 4) CMR-derived T1-Mapping with native T1Map (left) and ECV-Map for 3 slices (right) demonstrating an ECV of $31 \%$.

(C) HFpEF patients with ECV $\geq$ Median 1: Pressure-volume loops under transient preload reduction shows the steepest slope of the EDPVR line (green line). 2: Pressure-volume loops at baseline (yellow) and throughout excerise (red) with significant change in EDPVR (green points) 3: Echocardiographic Assement of Mitral valve inflow pattern (left) and septal mitral valve annular velocities (right) shows diastolic dysfunction without notable different to $B^{3}$. 4) CMR-derived T1-Mapping with native T1-Map (left) and ECV-Map for 3 slices (right) demonstrating an ECV of $36 \%$.

$\mathrm{CMR}=$ cardiac magnetic resonance, $\mathrm{ECV}=$ Extracellular Volume fraction, EDPVR=End-diastolic pressure volume relation, $\mathrm{HF}=$ Heart Failure, $\mathrm{HFpEF}=\mathrm{Heart}$ Failure with preserved ejection fraction, $\mathrm{LV}=$ left ventricular, $\mathrm{MV}=$ mitral valve.

Figure 1 Results of pressure volume analysis, echocardiography and CMR-imaging according to ECV group and controls. 